\title{
A NUMERICAL MODEL FOR THE 29-STRAND NET BRAID
}

E.M.J Niessen, L.J.M van de Klundert

University of Twente, Dept. of Applied Physics

P.O. Box 217, 7500 AE Enschede, The Netherlands

Abstract

A discrete modeling technique is used to analyze the 29-strand NET braid in changing magnetic fields. Input for the model is the $V-I$ relation of an individual strand and the contact resistance between touching strands. The model gives 3 dimensional current patterns, $V-I$ relations and corresponding energy losses for the cable. The analysis shows a strong dependence of the cable properties on the direction of the field.

\section{Introduction}

Properties of a superconducting cable are modeled by representing the cable as a network of node points ${ }^{1}$. Between two node points either flows a superconducting parallel current in a strand or a contact current between two touching strands. Kirchhoff's equations can be applied to the node points and the resulting meshes. The meshes incorporate the induced voltage due to applied magnetic field changes. Basic elements of the model are the $V-I$ relation of a single strand and the contact resistance between these strands.

Application of this, so called, network method was reported for simple cable geometries, e.g. a superconducting composite ${ }^{2}$, a round twisted cable and a Rutherford cable ${ }^{1,3}$. The complicated geometry of the 29 -strand NET braid, caused by the special braiding technique developed by $\mathrm{ABB}$, is modeled. The positions of the contact currents depend strongly on this braiding technique.

\section{Geometry of the cable}

We will explain the geometry of an idealized cable which is in good agreement with a realistic cable. The 29-strand NET braid has a cabling length $L_{c}$ of $460 \mathrm{~mm}$. Every $460 / 29 \approx 16$ $\mathrm{mm}$ the structure of a cross-section of the cable is repeated. This periodic length can again be divided into four equal parts of $4 \mathrm{~mm}$. When we project the positions of the strands of the cable on the cross-sectional plane $4 \times 29=116$ positions should occur. However, due to the crossing of the strands some positions will occur twice, and 97 points are left. These 97 points are shown in Figure 1. The letters A, B, C and D refer to the positions in the four subsequent planes. Here also the $x, y$ and $z$-direction are defined and the thickness $d$ of a strand is shown. The 19 points marked $E$ belong to both the $A$ and $\mathrm{C}$ plane. In this figure we can track the subsequent positions of one strand in one cabling length by following the letters A or $\mathrm{E}, \mathrm{B}, \mathrm{C}$ or $\mathrm{E}, \mathrm{D}, \mathrm{A}$ or $\mathrm{E}, \mathrm{B}, \ldots$. Recognize that these subsequent positions follow a complicated pattern in the crosssectional plane. Note that in the $\mathrm{A}$ and $\mathrm{C}$ plane the strands do not touch each other at all, whereas some do in the $B$ and $D$ plane. Defining all the contacts in the planes $B$ and D is a good approximation for the realistic cable.

Manuscript received September 24, 1990.

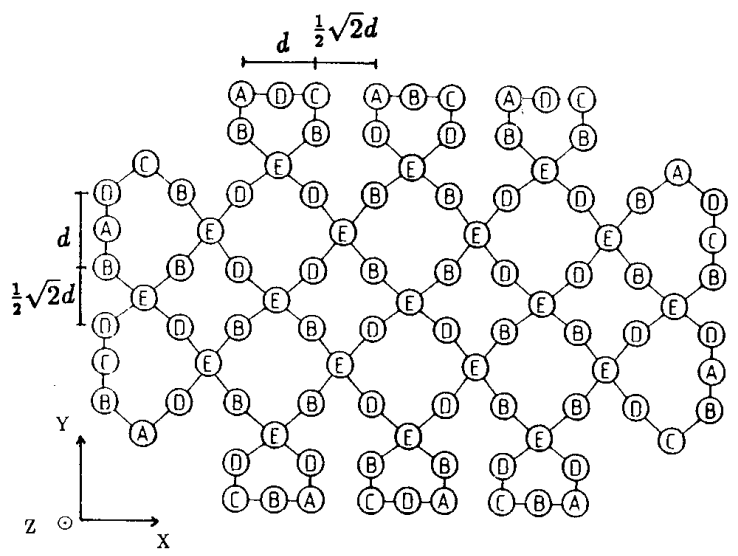

Figure 1: Positions of the strands at four equidistant planes subdividing one period of the braid. For A to E see text.

\section{The model}

In this section we define the currents in the model. One cabling length $L_{c}$ of the braid can be divided in 29 periods, each consisting of 2 layers. Layer 1 consists of a plane in which the strands do not touch (plane A) and a plane in which some strands can touch (plane B). For layer 2 they are plane $C$ and $D$ respectively. The positions of the strands in plane $B$ and $D$ for both layers are shown in Figures 2 and 3 respectively.

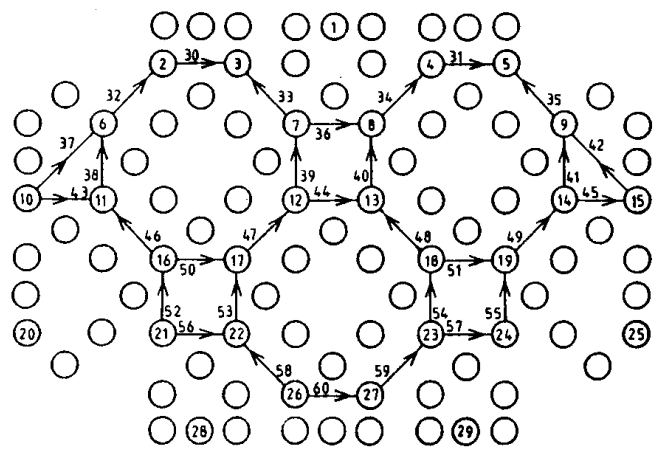

Figure 2: Definition of the parallel and contact currents in plane $B$ of Figure 1.

In these planes strands can touch each other, which causes contact currents to flow between these strands. In Figures 2 and 3 also the numbers of the parallel and contact currents in two subsequent layers are defined. Here the parallel currents are defined to flow in the positive $z$-direction and the contact currents flow in the direction given by the arrow. In one layer we see 29 parallel and 31 contact currents. The parallel currents are defined in the non-touching planes $A$ and $C$, while the contact currents are defined in the touching planes $B$ and $D$. This means that we have a network of node points connected by defined currents. In the calculations current $I_{10}$ and $I_{15}$ have a y-coordinate $d / 2$ greater and current $I_{75}$ and $I_{80}$ have a ycoordinate $d / 2$ smaller than shown in Figure 2 and 3. 


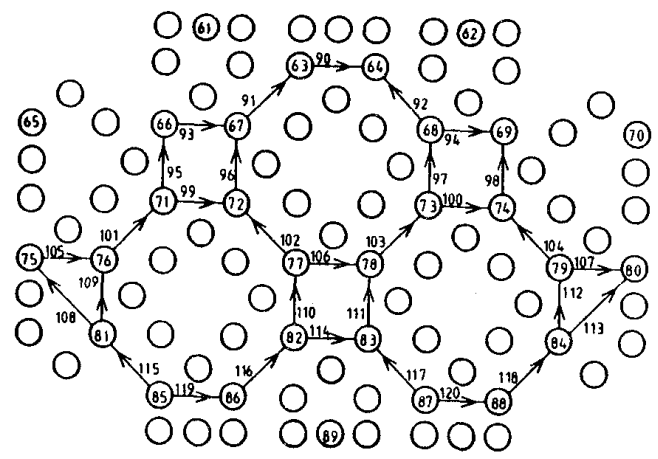

Figure 3: Definition of the parallel and contact currents in plane D of Figure 1.

In one period there are 120 unknown currents. In a piece of the braid of $M$ periods, $120 M(=N)$ unknowns must be determined.

\section{Equations}

For solving the currents we use Kirchhoff's equations together with a supplementary set of constitutive equations. We only consider time independent currents and do not take the induced field due to the currents themselves into account. Kirchhoff's equations read:

$$
\begin{gathered}
\sum_{h} I_{h}=0, \\
\sum_{i} V_{i}=-V^{i n d} .
\end{gathered}
$$

Eq.(1) is valid in each node point of the network. Index $h$ contains all the current numbers connected to a specific node point. Eq.(2) has a right hand side $-V^{\text {ind }}$, the flux change pointing through the surface of a mesh. Index $i$ contains all the voltage numbers in that specific mesh.

Once per period one has to add:

$$
\sum_{j} I_{j}=I_{0}
$$

stating that all currents pointing through some cross-section of the cable add up to the applied transport current $I_{0}$. The $I_{h}$ 's of (1) and the $V_{i}$ 's of (2) are connected through the constitutive equations. These equations can take three forms denoted by the indices $n, u$ and $s$ respectively ${ }^{1}$ :

- normal contact current:

$$
V_{n}=R_{k} I_{n}
$$

- unsaturated superconducting current if $\left|V_{u}\right| \leq V_{0}$ :

$$
V_{u}=\frac{V_{0} R_{p}}{I_{c} R_{p}+V_{0}} I_{u} \equiv R_{q} I_{u}
$$

- saturated superconducting current if $\left|V_{a}\right|>V_{0}$ :

$$
V_{\mathfrak{a}}=R_{p}\left(I_{\mathfrak{a}}-I_{c} \operatorname{sign}\left(V_{\mathfrak{\imath}}\right)\right) \text {. }
$$

In eq.(4-6) $R_{k}$ is the contact resistance, $R_{p}$ is the parallel resistance of the normal conducting part of a strand element, $R_{q}$ is the effective resistance of a strand element in the unsaturated case, $I_{c}$ is the threshold value of the saturation in a compos- ite superconducting strand, depending on its inner structure ${ }^{1}$, $V_{0}$ is the voltage across a strand element in case the element is just saturated $\left(V_{0}=8(0.460 / 58) R_{f}\left|\dot{B}_{\perp}\right| / 3 \pi\right), R_{f}$ is the radius of the superconducting filaments in a strand and $\dot{B}_{\perp}$ is the time derivative of the perpendicular component of the applied magnetic field. Substitution of (4-6) in (2) yields:

$$
\sum_{n} R_{k} I_{n}+\sum_{u} R_{q} I_{u}+\sum_{c} R_{p} I_{\bullet}=-V^{i n d}+\sum_{e} R_{p} I_{c} \operatorname{sign}\left(V_{\iota}\right) .
$$

Eqs.(1), (3) and (7) are used for the calculation of the parallel and contact currents. In one period we use 57 times eq.(1), once eq.(3) and 62 times eq.(7) for independent meshes. It is possible to minimize the maximal mesh length to three layers. We assume periodic boundary conditions over the total of $M$ periods. Because the maximal mesh length is 3 layers, these conditions are used only in the last period: for solving the currents in period $M$ we need currents in period $M+1$. These are set equal to the currents in period 1.

In the mesh equations the contact resistances satisfy eq.(4)

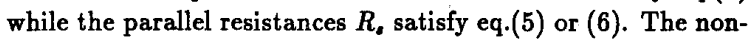
linearity in $R$, results in an iterative solving method. We assume the value of $R_{a}$ and the sign of $V_{G}$, and check the validity of the requirements on the resulting $V_{u}$ and $V$. For convergence the same restrictions can be used as described by Hartmann ${ }^{4}$, where it was shown that one should not allow a strand element to change from a negative saturation into a positive one or vice versa, but only allow saturated elements to turn in unsaturated ones or vice versa.

Recognize that the critical current $I_{c}$ can be taken to be $\dot{B}_{\perp}$ dependent ${ }^{1}$. This case is not considered here, because our first interest is to investigate the influence of the braiding technique on cable properties.

\section{$\underline{\text { Reduction technique }}$}

Considering the numbering of the currents, it is possible for this model to create a sparse and upper matrix, except for those equations in which the boundary conditions are included: the last period of the cable. The principal diagonal of this matrix is non-zero. We use equation $i$ of the matrix, with $i \in[1, N-120]$, for eliminating variable $I_{i}$ out of equation $j$, with $j \in[N-119, N]$. This results in a $120 \times 120$ full matrix for currents $N-119$ to $N$ (we prescribe the pivoting element for the elimination to be the principal diagonal). Solving the relatively small matrix gives the currents in the last period. Back substitution in equation $N-120$ to 1 renders the whole solution.

This technique gives an accurate solution because we use good pivots in the reduction of the matrix (for the mesh equations the principal diagonal $R_{k}$ is dominant in comparison to $R_{t}$ ) and a full pivoting matrix solver for the $120 \times 120$ matrix.

The operations for obtaining the whole solution can be divided into two parts:

1: the reduction: the mean number of non-zero coefficients in a row is $\alpha=5.4$. We eliminate $N-120$ variables from 120 equations.

2: full pivoting matrix solving: it uses $O\left(120^{3}\right)$ operations. The number of operations of the first step is linear proportional to the number of unknowns $N$ whereas the second does not depend on $N$. The technique reduces the CPU time of the program considerably in comparison to other sparse solvers. 


\section{Infinite periodic cables}

In this section we consider a cable with length $L$, consisting of $M$ periods in an applied magnetic field $\dot{B}$ in the $\mathrm{x}$ or $\mathrm{y}$ direction. The voltage across the cable per unit length is given by:

$$
V / l=\frac{1}{29 L} \sum_{i=1}^{M}\left\{\sum_{j=1}^{29} V_{i, j}+\sum_{j=61}^{89} V_{i, j}\right\},
$$

with $V_{i, j}$ is the voltage difference across a strand element $j$ in period $i$. The parallel and contact losses per cable volume are:

$$
\begin{aligned}
P_{\mathrm{par}} / \vartheta & =\frac{1}{\vartheta} \sum_{i=1}^{M}\left\{\sum_{j=1}^{29} I_{i, j} V_{i, j}+\sum_{j=61}^{89} I_{i, j} V_{i, j}\right\} \\
P_{k} / \vartheta & =\frac{1}{\vartheta} \sum_{i=1}^{M}\left\{\sum_{j=30}^{60} I_{i, j} V_{i, j}+\sum_{j=90}^{120} I_{i, j} V_{i, j}\right\},
\end{aligned}
$$

with $I_{i, j}$ current number $j$ in period $i$ and the cable volume $\vartheta=29 \pi\left(\frac{1}{2} d\right)^{2} L$.

For the unsaturated case $R_{0} \ll R_{k}$ which gives:

$$
\begin{aligned}
V / l & =c_{1} \frac{I_{0} R_{f} \dot{B}}{I_{c}}[V / m], \\
P_{p a r} / \vartheta & =c_{2} \frac{I_{0}^{2} R_{f} \dot{B}}{I_{c} d^{2}}+c_{3} \frac{R_{f} \dot{B}^{3} L_{c}^{2}}{I_{c} R_{k}^{2}}\left[W / m^{3}\right] \\
P_{k} / \vartheta & =c_{4} \frac{\dot{B}^{2} L_{c}}{R_{k}}\left[\mathrm{~W} / \mathrm{m}^{3}\right]
\end{aligned}
$$

for $R_{0}=V_{0} / I_{c}$ (as $R_{p} \gg V_{0} / I_{c}$ ). Some of the constants $c$ in the above expressions depend on the direction of the field. This is denoted by the extra subscript ' $x$ ' and ' $y$ ' for a $\dot{B}_{x}$ and $\dot{B}_{y}$ field respectively. The numerical calculated constants are: $c_{1 *}=c_{1 y}=2.9310^{-2}, c_{2 x}=c_{2 y}=1.2910^{-3}$ and

$$
\begin{array}{ll}
c_{3 x}=3.5310^{-3} ; & c_{3 y}=5.3210^{-3} ; \\
c_{4 x}=1.84 ; & c_{4 y}=6.01 .
\end{array}
$$

For a magnetic field in the $z$-direction we only give $P_{k} / \vartheta$ for $I_{0}=0$ :

$$
P_{k, z} / \vartheta=5.0810^{2} \frac{\dot{B}_{z}^{2} d^{2}}{R_{k} L_{c}}\left[W / m^{3}\right]
$$

For the saturated case $R$, is given by eq.(6), resulting in:

$$
\begin{aligned}
V / l & =\frac{58 R_{p} I_{c}}{L_{c}}\left(\frac{I_{0}}{29 I_{c}}-1\right)[\mathrm{V} / \mathrm{m}] \\
\text { and } P_{\mathrm{par}} / \vartheta & =\frac{I_{0}}{29 \pi d^{2} / 4} V / l\left[\mathrm{~W} / \mathrm{m}^{3}\right] .
\end{aligned}
$$

These two expressions are valid for both a $\dot{B}_{x}$ and $\dot{B}_{y}$ field for we neglected the induced losses. For the contact loss we get:

$$
P_{k} / \vartheta=\frac{\dot{B}^{2} L_{c}}{R_{k}} F\left[W / m^{3}\right]
$$

Here $F$ is a polynomial in $R_{k}$ and $R_{p}$ with coefficients depending on the direction of the field. We consider three cases:

$$
\begin{array}{lll}
R_{k} \gg R_{p}: & F_{x}=c_{4 x} ; & F_{y}=c_{4 y} ; \\
R_{k}=R_{p}: & F_{x}=2.6910^{-3} ; & F_{y}=2.0210^{-3} ; \\
R_{k} \ll R_{p}: & F_{z}=3.7110^{-3} R_{k}^{2} / R_{p}^{2} ; & F_{y}=4.3110^{-3} R_{k}^{2} / R_{p}^{2} .
\end{array}
$$

The partly saturated case will be analyzed numerically. When we start with a partly saturated configuration and $R_{k}$ decreases, $V$ ind will induce greater currents in a mesh, which means that
$S$, the relative number of saturated strand elements with respect to all strand elements, increases. Also $P_{p a r}$ and $P_{k}$ increase. When $R_{p}$ increases the resulting voltage will increase, because the cable has to carry a transport current $I_{0}: S$ and $P_{\text {par }}$ increase. $P_{k}$ decreases as the higher resistances in a mesh decrease the induced currents. When $R_{f}$ decreases $S$ increases. The employed parameter set is: $R_{k}=10^{-3} \Omega, R_{p}=10^{-8} \Omega$, $R_{f}=10^{-5} \mathrm{~m}, I_{\mathrm{c}}=500 \mathrm{~A}$ and $d=10^{-3} \mathrm{~m} . V / l, S$ and $P_{h} / \vartheta$ are given as function of $I_{0}$ for three values of $\dot{B}_{x}$ in Figure 4.
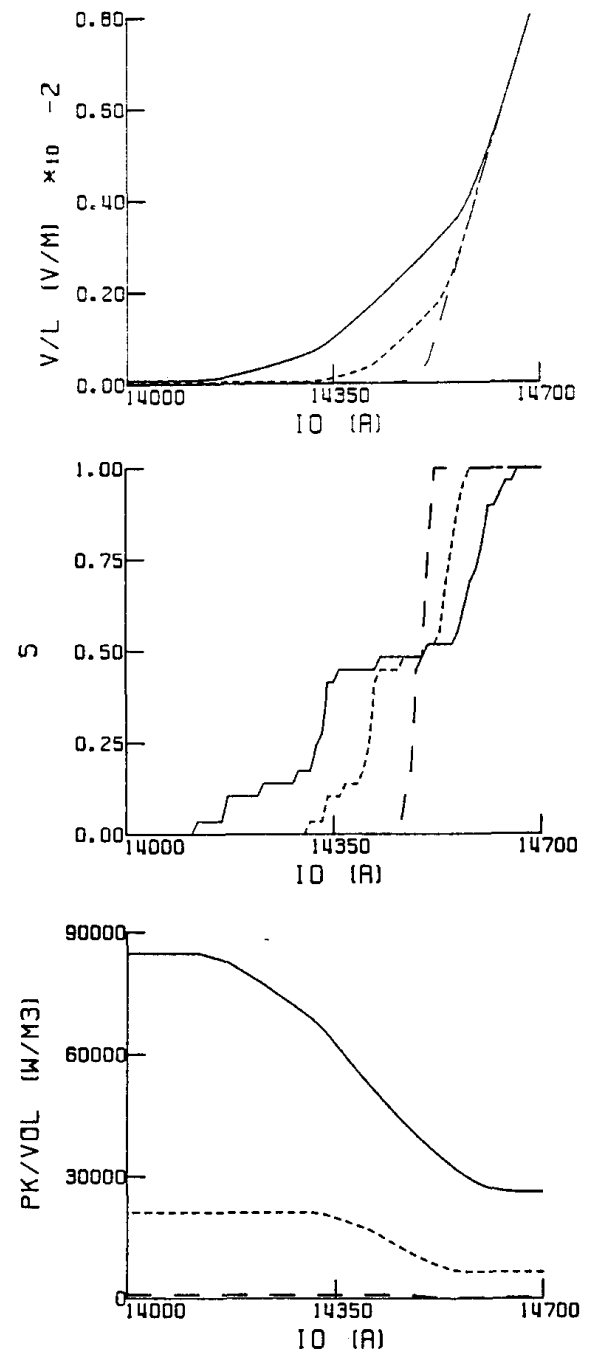

Figure 4: $V / l, S$ and $P_{k} / \vartheta$ of the cable as function of $I_{0}$. The lines (_- $),(--)$ and $\left(-{ }_{-}\right)$correspond to $\dot{B}_{x}=10,5$ and $1 T / s$ respectively.

The transition from an unsaturated to a completely saturated cable is given. Observe that $V / l$ consists of several, almost straight lines. Along such a line $S$ increases slowly, while at a change between two straight lines $S$ increases fast. We also see that due to the saturation $P_{k}$ decreases.

The saturation process described in the paragraph above is visualized in Figure 5. 


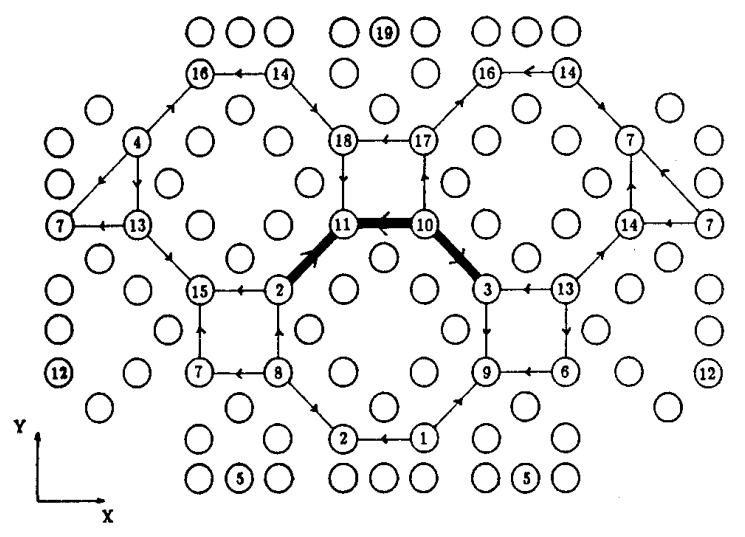

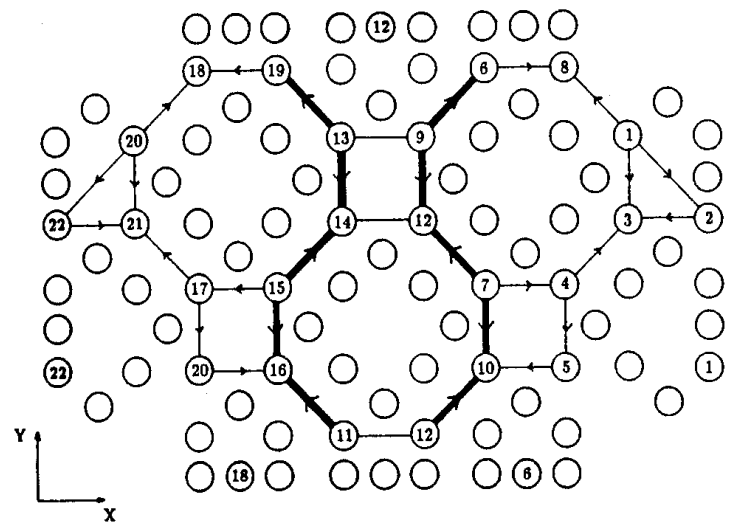

Figure 5: Saturation process in a $\dot{B}_{x}$ and $\dot{B}_{y}$ field (upper/lower figure) of $1 T / \mathrm{s}$ for increasing $I_{0}$. For the numbers see text.

It indicates the order in which the strands become saturated as $I_{0}$ increases from zero to its maximal value. In the upper and lower part we see the order for a $\dot{B}_{x}$ and $\dot{B}_{y}$ field of $1 \mathrm{~T} / \mathrm{s}$ respectively. For $I_{0}=0$ no saturation occurs. A higher number in the figure means that the strand saturates for a greater value of $I_{0}$. The arrows give the direction of the contact currents in case all the strands are saturated. The most important contact currents are indicated with a thick arrow: the absolute value of these contact currents is greater than $75 \%$ of the greatest contact current for that specific $\dot{B}$ field. We see that for the $\dot{B}_{y}$ field the contact currents are zero in the symmetrical line parallel to the $y$-axis in the middle of the cable, which is to be expected. Note that for a $\dot{B}_{y}$ field the number of important contact currents is much greater than for a $\dot{B}_{x}$ field.

\section{Cables of finite length}

Now we consider a finite piece of the braid with length $L$ placed in an applied magnetic field $B(t)=\dot{B} t$ with $\dot{B}$ constant. We investigate the steady state current distribution in the cable assuming that no saturation occurs. Due to the finite length of the cable the parallel currents at either end of the cable vanish. The resulting contact losses, scaled on their maximum are given as a function of $L / L_{c}$ for a $\dot{B}_{z}, \dot{B}_{y}$ and $\dot{B}_{z}$ field in Figure 6. As reference the scaled contact loss power density $p$ for a hollow, twisted cable is shown ${ }^{4,5}$, with the twistlength $L_{p}$ equal $L_{c}$;

$$
p=1-\left(\frac{L_{c}}{\pi L}\right)^{2} \sin ^{2}\left(\frac{\pi L}{L_{c}}\right),
$$

$P_{h, x}$ displays an oscillatory behaviour as a function of $L / L_{c}$ as does a twisted cable. $P_{k, y}$ oscillates with the double frequency $L / 2 L_{c}$. This doubling is caused by the symmetry of the model with respect to the centre line of the braid parallel to the $y$ axis. On this symmetrical axis the contact currents are equal to zero. The parallel currents crossing this line are not zero. $P_{k, z}$ is almost constant for $L>L_{c}$.

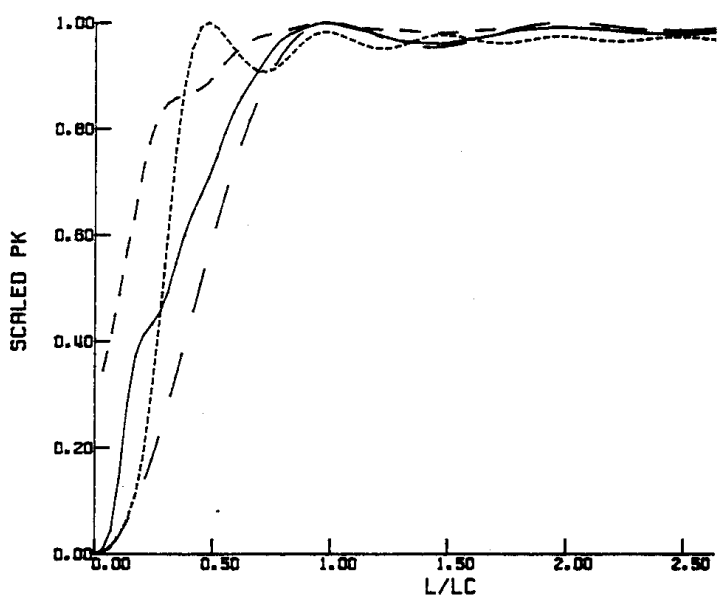

Figure 6: Scaled value of the contact losses as function of $L / L_{c}$. The lines (_ $),\left(--_{-}\right),\left(-_{-}\right)$and $\left({ }_{-}-\right)$correspond to a $\dot{B}_{x}, \dot{B}_{y}$ and $\dot{B}_{z}$ field on the braid and a $\dot{B}_{\perp}$ field on the hollow twist, respectively.

\section{References}

1. E.M.J. Niessen, D. ter Avest and L.J.M. van de Klundert, "Application of the network method to superconducting cables", Proc. LTEC90, July 1990, pp 5.4.

2. D. Ciazynski and B. Turck, "Theoretical and experimental study of the saturation of a superconducting composite under fast changing magnetic field", Cryogenics 24, Oct. 1984, pp 507-514.

3. V.E. Sytnikov et al., "Coupling losses in superconducting transposed conductors located in changing magnetic fields", Cryogenics 29, Sept. 1989, pp 926-930.

4. R.A. Hartmann, "A contribution to the understanding of AC losses in composite superconductors", thesis University of Twente, Enschede, 1989.

5. G. Ries and S. Takács, "Coupling losses in finite length of superconducting cables and in long cables partially in magnetic field", IEEE Transactions on Magnets 17, no. 5, 1981, pp 2281-2284. 